\title{
Optimal Error Bound and A Semi-discrete Difference Scheme for the Cauchy Problem of the Modified Helmholtz Equation
}

\author{
Ai-lin Qian ${ }^{1}$, Zi-min Chen ${ }^{2}$ \\ ${ }^{1}$ School of Mathematics and Statistics, Hubei University of Science and Technology, Xianning, \\ Hubei, 437100, People's Republic of China \\ ${ }^{2}$ School of Foundational Medicine, Hubei University of Science and Technology, Xianning, \\ Hubei, 437100, People's Republic of China
}

Keywords: III-posed Problems; The Cauchy Problem of Helmholtz Equation; Difference Schemes; Regularization

\begin{abstract}
The Cauchy problem of Helmholtz equation is severely ill-posed problem. In this paper, we consider the Cauchy problem for the Helmholtz equation where the Cauchy data is given at $X=0$ and the solution is sought in the interval $0<x<1$. A semi-discrete difference schemes together with a choice of regularization parameter is presented and error estimate is obtained. The numerical example shows the effectiveness of this method.
\end{abstract}

\section{Introduction}

The modified Helmholtz equation appears in many areas of engineering and science[1],such as in implicit marching schemes for the heat equation, in Debye-Huckel theory, in the linearization of the Poisson-Boltzmann equation and so on $[5,8,16]$. The direct problems for the modified Helmholtz equation have been studied extensively in the past century [19, 7]. However, in some practical problems, the boundary data on the whole boundary cannot be obtained. We only know the noisy data on a part of the boundary or at some interior points, giving rise to an inverse problem [6]. The Cauchy problem for the modified Helmholtz equation is an inverse problem and is typically ill-posed. It means that a small change in the Cauchy data may result a dramatic change in the solution. Therefore, it is necessary to study different highly efficient algorithms for solving it. In recent years, there are many special numerical methods to deal with this problem, such as the boundary element method[9], the method of fundamental solutions[10, 17], the conjugate gradient method[11], the Landweber method[9], quasi-reversibility and truncation method[14], quasi-boundary and Tikhonov type regularization method[13, 18], the Fourier regularization method [3, 4] and so on[15]. In the present paper we will consider the following problem with inhomogeneous Dirichlet data in a strip domain:

$$
\begin{aligned}
& u_{x x}+u_{y y}-k^{2} u=0,0<x<1,-\infty<y<\infty \\
& u(0, y)=\phi(y),-\infty<y<\infty \\
& u_{x}(0, y)=0,-\infty<y<\infty
\end{aligned}
$$

Where $k>0$ is a real number. We shall use a semi-discrete difference scheme to construct stable solution of the problem (1.1) and then obtain error estimate.

\section{Regularization for a Cauchy Problem of the Modified Helmholtz Equation}

Let $\hat{g}(\xi)$ denote the Fourier transform of function $g(x)$, which is defined by

$$
\hat{g}(\xi)=\frac{1}{\sqrt{2 \pi}} \int_{-\infty}^{\infty} e^{-i x \xi} g(x) d x
$$


The function $\phi(y), \phi^{\delta}(y) \in L^{2}(R)$ are the exact and measured data for problem (1.1) respectively, which satisfies

$$
\left\|\phi^{\delta}(\cdot)-\phi(\cdot)\right\| \leq \delta
$$

Where $\|\cdot\|$ denotes the $L^{2}-$ norm and constant $\delta>0$ is the noise level. Assume $u(x, \cdot) \in L^{2}(R)$ for all $0 \leq x<1$ and there holds the following a priori bound,

$$
\|u(1, \cdot)\| \leq E
$$

Where $E$ is a positive constant. It is easy to know that for problem (1.1), there holds

$$
\hat{u}(x, \xi)=\cosh \left(\sqrt{\xi^{2}+k^{2}} x\right) \hat{g}(\xi),
$$

and equivalently,

$$
u(x, y)=\frac{1}{\sqrt{2 \pi}} \int_{-\infty}^{\infty} e^{-i y \xi} \cosh \left(x \sqrt{|\xi|^{2}+k^{2}} \hat{\phi}(\xi)\right) d \xi
$$

Note that the fact $\cosh \left(x \sqrt{|\xi|^{2}+k^{2}}\right)$ is increases exponentially for $0<x<1$ as $|\xi \rightarrow \infty|$, the small disturbation for the data $\phi(x)$ will be amplified infinitely by this factor and lead to the integral (2.5) blow-up. Therefore, recovering the value $u(x, y)$ from the measured data $\phi^{\delta}(x)$ is severely ill-posed. To deal with this ill-pose problem, we introduce an regularization method. By using the central difference with step length $h$ to approximate the second derivative ${ }^{u_{y y}}$, we can get the following problem

$$
\begin{aligned}
& v_{x x}+\frac{v(x, y+h)-2 v(x, y)+v(x, y-h)}{h^{2}}-k^{2} u=0,0<x<1,-\infty<y<\infty \\
& v(0, y)=g_{\delta}(y),-\infty<y<\infty \\
& v_{x}(0, y)=0,-\infty<y<\infty .
\end{aligned}
$$

Taking Fourier transformation in the frequency space for problem (1.1), we can get

$$
\hat{v}(x, \xi)=\cosh \left[\frac{x}{h} \sqrt{\left(k^{2} h^{2}+2\right)-2 \cos (h \xi)}\right] \hat{g}_{\delta}(\xi)
$$

\section{Error Estimates}

Lemma2.1 Let

$$
p(\xi)=\frac{\cosh \left(\sqrt{\xi^{2}+k^{2} x}\right)}{\cosh \sqrt{\xi^{2}+k^{2}}}(\xi \geq 0), \text { then }
$$

(1) $p(\xi)$ is decrease as $\xi \geq 0$;

(2) $e^{-(1-x) \sqrt{\xi^{2}+k^{2}}} \leq p(\xi) \leq 2 e^{-(1-x) \sqrt{\xi^{2}+k^{2}}}$; 
(3)

$$
e^{\frac{x}{h} \sqrt{k^{2} h^{2}+4 \sin ^{2} \frac{h \xi}{2}}-\sqrt{\xi^{2}+k^{2} x}} \leq \frac{e^{\frac{x}{h} \sqrt{k^{2} h^{2}+4 \sin ^{2} \frac{h \xi}{2}}}+e^{-\frac{x}{h} \sqrt{k^{2} h^{2}+4 \sin ^{2} \frac{h \xi}{2}}}}{e^{\sqrt{\xi^{2}+k^{2} x}}+e^{-\sqrt{\xi^{2}+k^{2} x}}} \leq e^{\frac{x}{h} \sqrt{k^{2} h^{2}+4 \sin ^{2} \frac{h \xi}{2}}-\sqrt{\xi^{2}+k^{2} x}}
$$

Theorem 2.2 Suppose $v(x, y)$ is regularization solution for problem (1.1), whose Fourier Transformation is given by (2.7), $u(x, y)$ is exact solution for problem (1.1), whose Fourier transformation is given by (2.4), if $\operatorname{arccosh}\left(\frac{E}{\delta}\right)$, then we have the following error estimate

$$
\begin{aligned}
& h=\frac{2}{\arccos h\left(\frac{E}{\delta}\right)} \text {, then we have the following } \\
& 3) \text {, then }\|u(x, \cdot)-v(x, \cdot)\| \leq 2 E^{x}\left(\frac{\delta}{2}\right)^{1-x}(1+o(1)) .
\end{aligned}
$$

(2) If $\frac{E}{\delta}>\cosh (3)$, then

$$
\begin{aligned}
& \text { (2.1)for } 0<x<1-\frac{3 k}{2} \\
& \|u(x, \cdot)-v(x, \cdot)\| \leq E^{x}\left(\frac{\delta}{2}\right)^{1-x}(1+o(1))+\max \left\{\frac{9}{e^{3}(1-x)^{3}} \frac{E}{\left[\operatorname{arccosh}\left(\frac{E}{\delta}\right)\right]^{2}}, E^{x}\left(\frac{\delta}{2}\right)^{1-x}(1+o(1))\right\}
\end{aligned}
$$

(2.2) for $1-\frac{3 k}{2} \leq x<1$

$\|u(x, \cdot)-v(x, \cdot)\| \leq E^{x}\left(\frac{\delta}{2}\right)^{1-x}(1+o(1))+\max \left\{\frac{x \operatorname{arccosh}\left(\frac{E}{\delta}\right)}{3} e^{-(1-x) \arccos h\left(\frac{E}{\delta}\right)}, E^{x}\left(\frac{\delta}{2}\right)^{1-x}(1+o(1))\right\}$.

Proof. Acording Parseval formula and (2.2),(2.3), we can get $\left.\|u(x, ;)-v(x, \dot{)})\|=\|\hat{u}(x, \cdot)-\hat{v}(x)\|=,\| \cosh \left(\sqrt{\xi^{2}+k^{2}} x\right) \hat{g}(\xi)-\hat{g}_{\delta}(\xi) \cosh \frac{x}{h} \sqrt{\left(k^{2} h^{2}+2\right)-2 \cos (h \xi)}\right] \|$ $\left.\leq\left\|\frac{\cosh \left(\sqrt{\xi^{2}+k^{2}} x\right)-\cosh \left[\frac{x}{h} \sqrt{\left(k^{2} h^{2}+2\right)-2 \cos (h \xi)}\right]}{\cosh \sqrt{\xi^{2}+k^{2}}} \hat{f}(\xi)\right\|+\| \cosh \frac{x}{h} \sqrt{\left(k^{2} h^{2}+2\right)-2 \cos (h \xi)}\right]\left(\hat{g}(\xi)-\hat{g}_{\delta}(\xi)\right) \|$ $\leq \sup _{\xi \in R} A(\xi) E+\sup _{\xi \in R} B(\xi) \delta$.

where

$$
\begin{array}{r}
A(\xi)=\frac{\cosh \left(\sqrt{\xi^{2}+k^{2}} x\right)-\cosh \left[\frac{x}{h} \sqrt{\left(k^{2} h^{2}+2\right)-2 \cos (h \xi)}\right]}{\cosh \sqrt{\xi^{2}+k^{2}}} \\
B(\xi)=\cosh \left[\frac{x}{h} \sqrt{\left(k^{2} h^{2}+2\right)-2 \cos (h \xi)}\right.
\end{array}
$$

Firstly we can estimate $B(\xi)$ as 


$$
B(\xi)=\leq \cosh \left(\frac{x}{h} \sqrt{k^{2} h^{2}+4}\right) \leq \cosh \left(\frac{2 x}{h}\right) .
$$

It is easy to know that the asymptotic expression of $B(\xi) \delta$ is

$$
B(\xi) \delta \leq \cosh \left(\frac{2 x}{h}\right) \delta=E^{x}\left(\frac{\delta}{2}\right)^{1-x}(1+o(1)), \delta \rightarrow 0
$$

Because $A(\xi)$ is a even function, then

Case 1. If $\sqrt{\xi^{2}+k^{2}} \geq \xi_{0}=\frac{2}{k}=\arccos h\left(\frac{E}{\delta}\right)$, from $\sqrt{\xi^{2}+k^{2}} x \leq \frac{x}{h} \sqrt{k^{2} h^{2}+4 \sin ^{2} \frac{h \xi}{2}}$, we have

$$
\frac{e^{\frac{x}{h}} \sqrt{k^{2} h^{2}+4 \sin ^{2} \frac{h \xi}{2}}+e^{-\frac{x}{h}} \sqrt{k^{2} h^{2}+4 \sin ^{2} \frac{h \xi}{2}}}{e^{\sqrt{\xi^{2}+k^{2}} x}+e^{-\sqrt{\xi^{2}+k^{2} x}}} \leq 1
$$

from Lemma 2.1, we have

$$
\begin{aligned}
& A(\xi) E \leq \frac{\cosh \left(\sqrt{\xi^{2}+k^{2} x}\right)}{\cosh \sqrt{\xi^{2}+k^{2}}} E \leq \frac{\cosh \left(\frac{2 x}{h}\right)}{\cosh \left(\frac{2}{h}\right)} E \\
& =\delta \cosh \left(x \arccos \left(\frac{E}{\delta}\right)\right)=E^{x}\left(\frac{\delta}{2}\right)^{1-x}(1+o(1)), \delta \rightarrow 0 .
\end{aligned}
$$

Case 2. if $\sqrt{\xi^{2}+k^{2}}<\xi_{0}=\frac{2}{h}=\arccos h\left(\frac{E}{\delta}\right)$, from Lemma 2.1,we have

$$
A(\xi) E \leq 2 e^{-(1-x) \sqrt{\xi^{2}+k^{2}}}\left(1-e^{\frac{x}{h} \sqrt{k^{2} h^{2}+4 \sin ^{2} \frac{h \xi}{2}}-\sqrt{\xi^{2}+k^{2} x}}\right) E .
$$

By using the inequality $1-y \leq e^{-y}(y>0)$, we get

$$
A(\xi) E \leq E \frac{2 x}{h} e^{-(1-x) \sqrt{\xi^{2}+k^{2}}}\left[h \sqrt{\xi^{2}+k^{2}}-2\left|\sin \frac{h \sqrt{\xi^{2}+k^{2}}}{2}\right|\right]
$$

Let $r=\frac{h \sqrt{\xi^{2}+k^{2}}}{2}$, for $h \sqrt{\xi^{2}+k^{2}}<2$, we have

$$
A(\xi) E \leq E \frac{4 x}{h} e^{\frac{2(x-1) r}{h}}(r-|\sin r|)
$$

From $|\sin r| \geq r-\frac{r^{3}}{6}(r>0)$, we have 


$$
A(\xi) E \leq E \frac{4 x}{h} e^{\frac{2(x-1) r}{h}} \frac{r^{3}}{6}=\frac{2 x}{3 h} m(r, x),
$$

Where $m(r, x)=e^{\frac{2(x-1) r}{k}} r^{3}$, from $\frac{\partial m}{\partial r}=e^{\frac{2 r(x-1)}{h}} r^{2}\left(3-\frac{2 r(1-x)}{h}\right)$, we know that $r_{0}=\frac{3 h}{2(1-x)}$ is the extreme point of function $m(r, x)$.If $r_{0} \leq 1$, then

$$
m(r, x) \leq m\left(r_{0}, x\right)=e^{\frac{-2 r_{0}(1-x)}{h}} r_{0}^{3}=e^{-3}\left(\frac{3 h}{2(1-x)}\right)^{3}
$$

$$
A(\xi) E \leq \frac{2 x}{3 h} e^{\frac{-2 r_{0}(1-x)}{h}} r_{0}^{3} E=\frac{9}{e^{3}(1-x)^{3}} \frac{E}{\left[\arccos h\left(\frac{E}{\delta}\right)\right]^{2}} \rightarrow 0
$$

If $r_{0}>1$, then the extreme point of function $m(r, x)$ is $r=1$, so

$$
A(\xi) E \leq \frac{2 x}{3 h} m(1, x) E=\frac{x \arccos h\left(\frac{E}{\delta}\right)}{3} e^{-(1-x) \arccos h\left(\frac{E}{\delta}\right)} E \rightarrow 0 .
$$

We can conclude that the following hold,

(1) if $\frac{E}{\delta}<\cosh (3)$, then for $x \in(0,1), r_{0}=\frac{3 h}{2(1-x)}>1$, combining (3.8) and (3.16), we have

$$
A(\xi) E \leq \max \left\{\frac{x \arccos h\left(\frac{E}{\delta}\right)}{3} e^{-(1-x) \arccos h\left(\frac{E}{\delta}\right)} E, E^{x}\left(\frac{\delta}{2}\right)^{1-x}(1+o(1))\right\}
$$

Because $\arccos h(x)=\ln \left(x+\sqrt{x^{2}-1}\right)$, we have the following asymptotic expression

$$
\begin{aligned}
& e^{-(1-x) \operatorname{arccosh}\left(\frac{E}{\delta}\right)} E=e^{-(1-x)\left(\ln \left(\frac{E}{\delta}\right)+\sqrt{\left.\left(\frac{E}{\delta}\right)^{2}-1\right)}\right.}=E^{x}\left(\frac{\delta}{2}\right)^{1-x}(1+o(1)), \delta \rightarrow 0 . \\
& \text { So } \quad\|u(x, \cdot)-v(x, \cdot)\| \leq 2 E^{x}\left(\frac{\delta}{2}\right)^{1-x}(1+o(1))
\end{aligned}
$$

(2) if $\frac{E}{\delta}>\cosh (3)$ then

(2.1) if $0<x<1-\frac{3 h}{2}$, then $r_{0}<1$, so for $h \sqrt{k^{2}+\xi^{2}}<2$, combining (3.15) and (3.8), we have

$$
A(\xi) E \leq \max \left\{\frac{9}{e^{3}(1-x)^{3}} \frac{x E}{\left[\arccos h\left(\frac{E}{\delta}\right)\right]^{2}}, E^{x}\left(\frac{\delta}{2}\right)^{1-x}(1+o(1))\right\}
$$


Similarly, we have

$$
\|u(x, \cdot)-v(x, \cdot)\| \leq E^{x}\left(\frac{\delta}{2}\right)^{1-x}(1+o(1))+\max \left\{\frac{9}{e^{3}(1-x)^{3}} \frac{E}{\left[\operatorname{arccosh}\left(\frac{E}{\delta}\right)\right]^{2}}, E^{x}\left(\frac{\delta}{2}\right)^{1-x}(1+o(1))\right\} .
$$

(2.2) if $1-\frac{3 h}{2} \leq x<1$, then $r_{0} \geq 1$, so for $h \sqrt{k^{2}+\xi^{2}}<2$, combining (3.15) and (3.8), we have the following error estimate

$$
\|u(x, \cdot)-v(x, \cdot)\| \leq E^{x}\left(\frac{\delta}{2}\right)^{1-x}(1+o(1))+\max \left\{\frac{x \arccos h\left(\frac{E}{\delta}\right)}{3} e^{-(1-x) \operatorname{arccosh}\left(\frac{E}{\delta}\right)}, E^{x}\left(\frac{\delta}{2}\right)^{1-x}(1+o(1))\right\}
$$

Remark. It is easy to see that the space step length $\$ \mathrm{~h} \$$ is the regularization parameter of this problem. In the conclusion, we give a rule for choosing the parameter, which is very important for the study of ill-posed problems.

\section{Numerical Test}

In this section, a simple numerical example is devised to verify the validity of the proposed method.

We consider the following Cauchy problem:

$$
\begin{aligned}
& \Delta u(x, y)-k^{2} u(x, y)=0,0<x<\pi, 0<y<1, \\
& u(x, 0)=\phi(x)=\sum_{n=1}^{\infty} c_{n} \sin n x, 0 \leq x \leq \pi, \\
& u_{y}(x, 0)=h(x)=0,0 \leq x \leq \pi, \\
& u(0, y)=u(\pi, y)=0,0 \leq y \leq 1 .
\end{aligned}
$$

Take $u(x, y)=\sin x \cosh \left(\sqrt{1+k^{2}} y\right)-2 \sin (2 k x) \cosh (\sqrt{5} k y)$ as the exact solution for the Cauchy problem (4.1). The Cauchy data $\phi(x)=\sin x-\sin 2 k x$. Suppose the vector $\Phi$ represents samples from the function $\phi$. The noisy discrete data function is generated by adding random errors to the exact data function, at every grid point, i.e.,

$$
\Phi_{\delta}(n)=\Phi(n)+\varepsilon_{n},\left|\varepsilon_{n}\right| \leq \delta, n=0,1, \mathrm{~L}, N_{t}
$$

Where the $\varepsilon_{n}$ 's are independent random Gaussian variables with variance $\sigma^{2}=\varepsilon^{2}$. In the following, the capital letter is used to denote the samples of the corresponding function. We implement the algorithm in Matlab 7.10. The relative weighted $l^{2}$ errors for the recovered function are calculated as

$$
\frac{\left[\frac{1}{N+1} \sum_{n=0}^{N}\left|U_{\alpha}^{\delta}(n, y)-U(n, y)\right|\right]^{\frac{1}{2}}}{\left[\frac{1}{N+1} \sum_{n=0}^{N}|U(n, y)|^{2}\right]^{\frac{1}{2}}}
$$


Where $N$ is the number of grid in the interval ${ }^{[0, \pi]}$. Figure 1-3 demonstrate the computation effects at $y=1,0.5,0.1$,respectively. From the results in figure 1-3, we see that it is better when ${ }^{y}$ is closer to $y=0$.For large noise level $\delta=0.1$, the results are given as in Fig.4, which also displays good approximation.
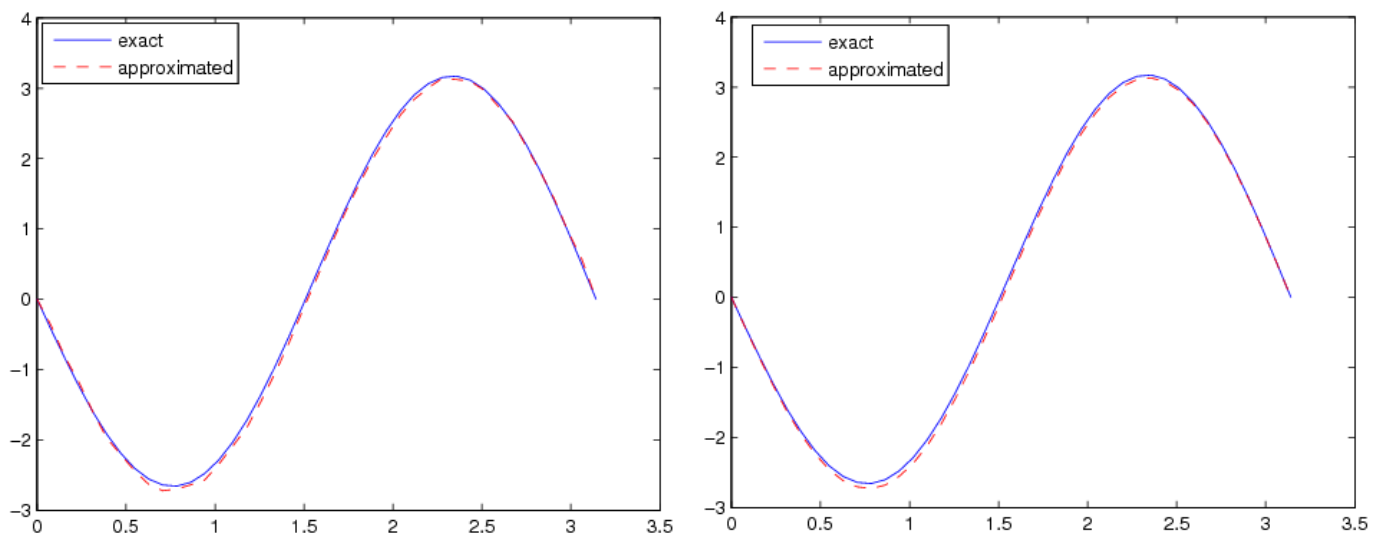

Figure1:(a): $y=1, \delta=0.01, \alpha=0.13, k=1$. (b): $y=1, \delta=0.001, \alpha=0.13, k=1$.
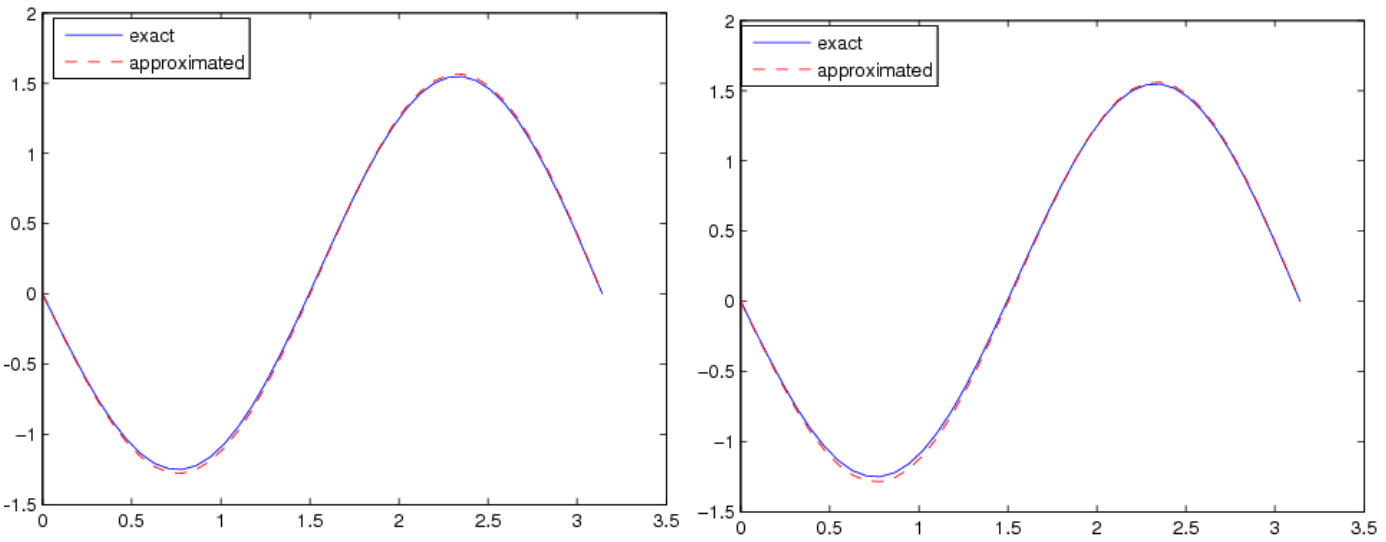

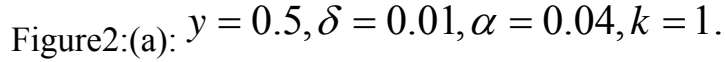

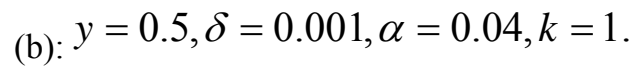
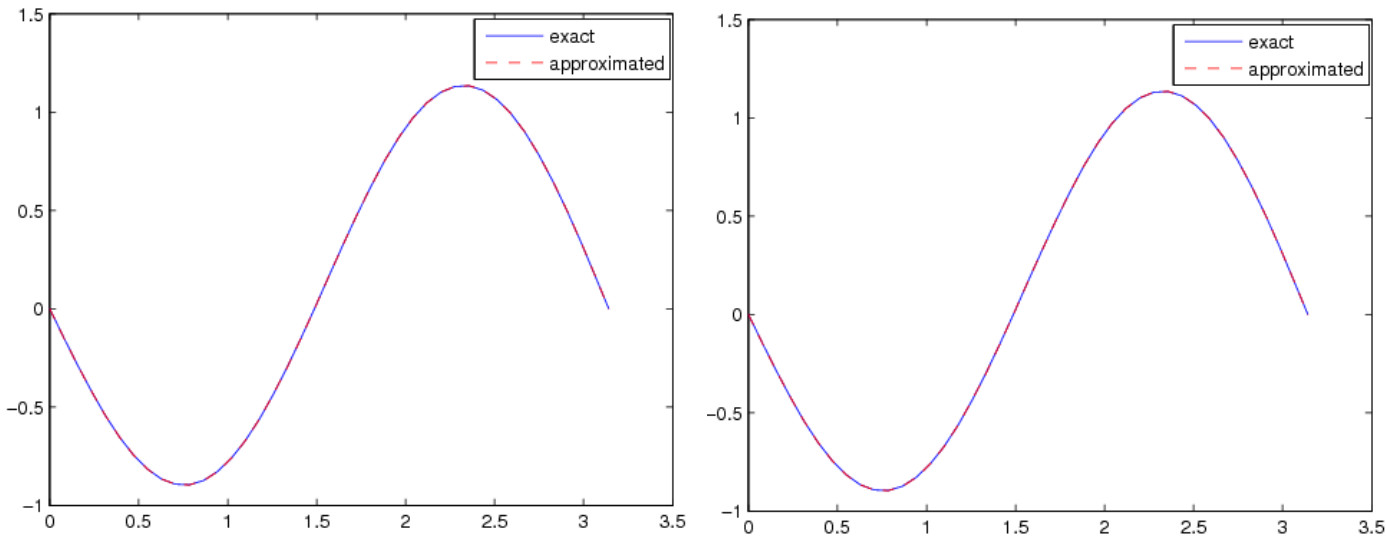

Figure3:(a): $y=0.1, \delta=0.01, \alpha=0.002, k=1$. (b): $y=0.1, \delta=0.001, \alpha=0.002, k=1$. 

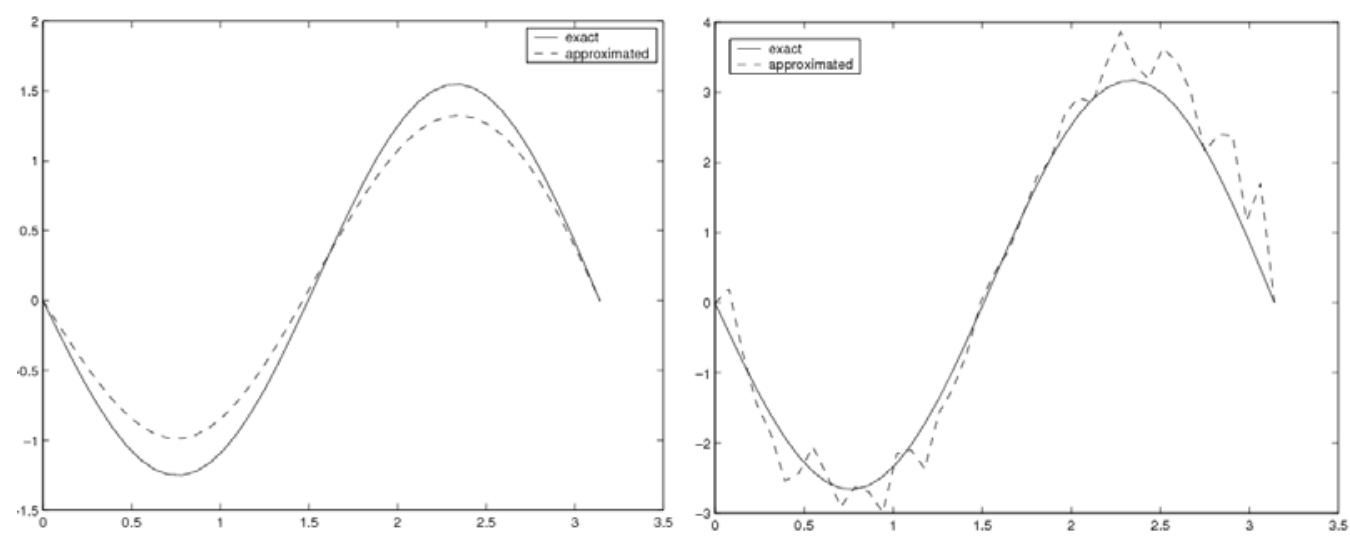

Figure4:(a): ${ }^{y=0.5, \delta=0.10, \alpha=0.10, k=1}$

(b): $y=1, \delta=0.1, \alpha=0.10, k=1$.

\section{Acknowledgement}

This research was financially supported Supported by Natural Science Foundation of Hubei Province of China(2015CFB708), Educational Commission of Hubei Province of China(D20152801), and the NNSF of China(No.71473073)

\section{References}

[1]H.W. Cheng, J.F. Huang and T.J. Leiterman, An adaptive fast solver for the modified Helmholtz equation in two dimensions, J. Comput. Phys. 211(2):(2006), 616-637.

[2]H.W. Engl, M. Hanke and A. Neubauer, Regularization of Inverse Problems, Kluwer Academic Publishes, Boston, 1996.

[3]C.L. Fu, X.L. Feng and Z. Qian, The Fourier regularization for solving the Cauchy problem for the Helmholtz equation, Appl. Numer. Math., 59(10): (2009), 2625-2640.

[4]C.L. Fu and Z. Qian, Numerical pseudodifferential operator and Fourier regularization, Adv. Comput. Math., 33(4), (2010), 449-470.

[5]A.H. Juffer, E.F.F. Botta, B.A.M.V. Keulen, A.V.D. Ploeg and H.J.C. Berendsen, The electric potential of a macromolecule in a solvent: a fundamental approach, $\mathrm{J}$. Comput. Phys., 97(1):(1991) 144-171.

[6] J. Hadamard, Lectures on Cauchy's Problem in Linear Partial Differential Equations, Dover Publications, New York, 1953.

[7] X. Li, On solving boundary value problems of modified Helmholtz equations by plane wave functions, J. Comput. Appl. Math., 195(1):(2006) 66-82.

[8]J. Liang and S. Subramaniam, Computation of molecular electrostatics with boundary element methods, Biophys. J. 73(4):(1997) 1830-1841.

[9]L. Marin, L. Elliott, P.J. Heggs, D.B. Ingham, D. Lesnic and X. Wen, BEM solution for the Cauchy problem associated with Helmholtz-type equations by the Landweber method, Eng. Anal. Bound. Elem., 28 (9) (2004), 1025-1034. 
[10] L. Marin and D. Lesnic, The method of fundamental solutions for the Cauchy problem associated with two-dimensional Helmholtz-type equations, Comput. Struct., 83 (45) (2005), 267-278.

[11] L. Marin, L. Elliott, P.J. Heggs, D.B. Ingham, D. Lesnic, X.Wen, Conjugate gradientboundary element solution to the Cauchy problem for Helmholtz-type equations, Comput. Mech., 31(3-4):(2003), 367-377.

[12]M.T. Nair, E. Schock and U. Tautenhahn, Morozov's discrpancy principle under general source conditions, Z. Anal. Anw., 22(1)(2003), 199-214.

[13] H.H. Qin and D.W. Wen, Tikhonov type regularization method for the Cauchy problem of the modified Helmholtz equation, Appl. Math. Comput., 203:(2008), 617-628. [14] H.H. Qin and T. Wei, Quasi-reversibility and truncation methods to solve a Cauchy problem for the modified Helmholtz equation, Math Comput Simul., 80 (2) (2009) 352-366.

[15]T. Reginska and U. Tautenhahn, Conditional stability estimates and regularization with applications to Cauchy problems for the Helmholtz equation, Numer. Funct. Anal. and Optimiz., 30 (2009), 1065 C1097.

[16] W.B. Russell, D.A. Sville and W.R. Schowalter, Colloidal Dispersions, Cambridge University Press, Cambridge, 1991.

[17] T.Wei, Y. Hon, L. Ling, Method of fundamental solutions with regularization techniques for Cauchy problems of elliptic operators, Eng. Anal. Boundary Elem., 31(4):(2007), 373-385.

[18]X.T. Xiong, W.X. Shi and X.Y. Fan, Two numerical methods for a Cauchy problem for modified Helmholtz equation, Appl. Math. Modell., 35(2011): 4951-4964. [19]A. Yoneta, M. Tsuchimoto, T. Honma, Analysis of axisymmetric modified Helmholtz equation by using boundary element method, IEEE Trans. Magn. 26 (2) (1990) $1015 \mathrm{C} 1018$. 